\title{
Effects of Schisandra chinensis Turcz. fruit on contact dermatitis induced by dinitrofluorobenzene in mice
}

\author{
HEE JUNG LEE ${ }^{1}$, SUZY JO ${ }^{2}$, JEONGHYUN RYU ${ }^{2}$, HAN-SOL JEONG ${ }^{3}$, GUEMSAN LEE ${ }^{4}$, \\ MI HEON RYU ${ }^{5}$, MYEONG HO JUNG ${ }^{1}$, HYUNGWOO KIM ${ }^{2}$ and BYUNG JOO KIM ${ }^{1}$ \\ Divisions of ${ }^{1}$ Longevity and Biofunctional Medicine, ${ }^{2}$ Pharmacology and ${ }^{3}$ Applied Medicine, \\ Pusan National University School of Korean Medicine, Yangsan, Gyeongsangnam-do 626-870; \\ ${ }^{4}$ Department of Herbology, Wonkwang University College of Medicine, Iksan, North Jeolla 570-749; \\ ${ }^{5}$ Department of Oral Pathology, Pusan National University School of Dentistry, \\ Yangsan, Gyeongsangnam-do 626-870, Republic of Korea
}

Received July 8, 2014; Accepted March 24, 2015

DOI: $10.3892 / \mathrm{mmr} .2015 .3618$

\begin{abstract}
Schisandra chinensis Turcz. fruit is widely used to treat skin diseases. The aim of this study was to determine the anti-inflammatory effects of the methanol extract of $S$. chinensis (MESC) on 1-fluoro-2,4-dinitrofluorobenzene (DNFB)-induced contact dermatitis (CD) in mice. The effects of MESC on ear thickness and weight, histopathological changes, immune cell filtration and cytokine production were investigated in DNFBinduced CD mice. Topical application of MESC effectively inhibited ear swelling (30 or $300 \mu \mathrm{g}$ on the left ear, $\mathrm{P}<0.001$; $30 \mu \mathrm{g}$ on the right ear, $\mathrm{P}<0.001)$. MESC also inhibited hyperplasia, spongiosis (100 $\mu$ g/ear, $\mathrm{P}<0.05$ and $300 \mu \mathrm{g} / \mathrm{ear}, \mathrm{P}<0.001$, respectively) and immune cell infiltration (100 $\mu \mathrm{g} / \mathrm{ear}, \mathrm{P}<0.05$; $300 \mu \mathrm{g} / \mathrm{ear}, \mathrm{P}<0.001)$ induced by DNFB. In addition, MESC suppressed increases in tumor necrosis factor (TNF)- $\alpha$ levels (100 or $300 \mu \mathrm{g} / \mathrm{ear}, \mathrm{P}<0.05)$, interferon (INF)- $\gamma(30 \mu \mathrm{g} / \mathrm{ear}$, $\mathrm{P}<0.05 ; 100 \mu \mathrm{g} / \mathrm{ear}, \mathrm{P}<0.01 ; 300 \mu \mathrm{g} / \mathrm{ear}, \mathrm{P}<0.001)$, interleukin (IL)-6 (300 $\mu \mathrm{g} / \mathrm{ear}, \mathrm{P}<0.05)$ and monocyte chemoattractant protein (MCP)-1 (30 $\mu \mathrm{g} / \mathrm{ear}, \mathrm{P}<0.05 ; 100 \mu \mathrm{g} / \mathrm{ear}, \mathrm{P}<0.01 ; 300 \mu \mathrm{g} /$ ear, $\mathrm{P}<0.001)$. These results suggest that the anti-inflammatory effects of MESC are mediated by the reduced production of TNF- $\alpha$, IFN- $\gamma$, IL-6 and MCP-1, and that MESC has potential use for the treatment of inflammatory skin diseases.
\end{abstract}

Correspondence to: Professor Hyungwoo Kim, Division of Pharmacology, Pusan National University School of Korean Medicine, 49 Busandaehakro, Yangsan, Gyeongsangnam-do 626-870, Republic of Korea

E-mail: kronos7@pusan.ac.kr

Professor Byung Joo Kim, Division of Longevity and Biofunctional Medicine, Pusan National University School of Korean Medicine, 49 Busandaehakro, Yangsan, Gyeongsangnamdo 626-870, Republic of Korea

E-mail: vision@pusan.ac.kr

Key words: Schisandra chinensis, traditional Chinese medicine, inflammation, contact dermatitis

\section{Introduction}

Schisandra chinensis is a member of the family Schisandraceae and the genus Schisandra. This climbing plant is widely distributed in the Russian Far East, China, Japan and Korea, and is often used to treat asthenia, cough, asthma and exhaustion $(1,2)$. In addition, it is widely used to treat skin diseases, such as, atopic dermatitis, photo-aging and hair loss (3-6). Recently, $S$. chinensis has been reported to aid liver regeneration, inhibit hepatocarcinogenesis, exhibit antioxidant activity, restore blood sugar balance, reduce blood pressure, and to exhibit anti-inflammatory, wound healing and antitumor effects $(2,7,8)$.

Contact dermatitis (CD) is one of the most common occupational skin diseases (9). Furthermore, its timely and accurate diagnosis is important for achieving satisfactory outcomes (9). Primary treatment for $\mathrm{CD}$ requires avoidance of contact with the offending agent, which is often difficult in cases of occupational CD (10). As a result, treatment for CD tends to consist of the repeated administration of immune-modulatory and anti-inflammatory agents, such as corticosteroids. However, although they are highly effective when used to treat allergic and inflammatory diseases, the dosages and treatment schedules of corticosteroids are frequently restricted due to serious potential side effects (10), which minimize their use, particularly when continuous application is required. Conversely, traditional medicines have been used in Korea as alternatives and to complement corticosteroids as they are safe and inexpensive (11).

However, the use of $S$. chinensis to treat $\mathrm{CD}$ with or without corticosteroids has not been fully investigated. Therefore, in the present study, the anti-inflammatory effects of S. chinensis was evaluated using a mouse model of CD. In particular, the effects of S. chinensis on ear thickness, ear weight, histopathological changes in ear tissue, and cytokine levels in inflamed tissues were assessed in vivo.

\section{Materials and methods}

Chemicals and reagents. 1-Fluoro-2,4-dinitrofluorobenzene (DNFB), dimethyl sulfoxide (DMSO) and dexamethasone (DEX/PLGA) were purchased from Sigma-Aldrich (St. Louis, 
MO, USA). Protein extraction kits were obtained from Intron Bio (Daejeon, Korea) and cytometric bead array kits were acquired from BD Biosciences (Franklin Lakes, NJ, USA).

Preparation of the methanol extract of S. chinensis (MESC). Ripe $S$. chinensis fruits were purchased from Hwalim Medicinal Herbs (Pusan, Korea). The extraction was performed using a standard procedure (8). Briefly, $50 \mathrm{~g}$ ripe S. chinensis fruits were immersed in 1,000 $\mathrm{ml}$ methanol, sonicated for $30 \mathrm{~min}$, and agitated for $24 \mathrm{~h}$. The extract was then filtered through Whatman filter paper (no. 20; VWR International, LLC, Arlington Heights, IL, USA), evaporated under reduced pressure using a vacuum evaporator (Eyela, Tokyo, Japan), and lyophilized using a freeze dryer (Labconco, Kansas City, MO, USA). Finally, 15.9 g lyophilized powder (MESC) was obtained (yield, 31.8\%). A sample of MESC (voucher no. MH2010-010) was deposited at the Division of Pharmacology, School of Korean Medicine, Pusan National University (Yansan, Korea).

Animals. Male 6 week-old BALB/c mice were purchased from Samtaco (Incheon, Korea) and housed under specific pathogen-free conditions with a $12 \mathrm{~h}$ light/dark cycle and free access to standard rodent food and water. The animals were sacrificed by cervical dislocation. All animal experiments were approved by the Pusan National University Animal Care and Use Committee and conducted according to institutional guidelines (PNU-2011-000406).

Induction of $C D$ and experimental design. $\mathrm{CD}$ induction was performed using DNFB. Briefly, mice were sensitized by applying $50 \mu \mathrm{l} \mathrm{DNFB}(0.1 \%, \mathrm{v} / \mathrm{v})$ in acetone and olive oil (AOO, 4:1) onto shaved backs for three consecutive days. Four days after sensitization, each mouse was challenged by applying $30 \mu \mathrm{l}$ DNFB $(0.2 \%$, v/v) in AOO onto the dorsum of both ears on alternate days. For topical applications, MESC and DEX were dissolved in ethanol, filtered using a $0.45-\mu \mathrm{m}$ syringe filter and finally diluted in AOO. MESC in solution (30, 100 or $300 \mu \mathrm{g} / \mathrm{ear}$ ) was applied onto the dorsum of both ears daily for six consecutive days. Naïve animals (NOR) were neither sensitized nor challenged with DNFB $(n=6)$. Control animals (CTL) were sensitized and challenged with DNFB in $\mathrm{AOO}$, and then vehicle was applied (ethanol and AOO) $(n=9)$. MESC treated animals were sensitized and challenged with DNFB, and then administered with 30,100 or $300 \mu \mathrm{g} / \mathrm{ear}$ MESC ( $n=9)$. DEX treated animals were sensitized and challenged with DNFB and then $75 \mu \mathrm{g} /$ ear DEX in ethanol and $\mathrm{AOO}(\mathrm{n}=6)$ was applied as a positive control. The experimental design is summarized in Fig. 1.

Measurement of ear thickness and weight. Mice were anesthetized with $30 \mathrm{mg} / \mathrm{kg}$ Zoletil (Virbac, Carros, France) and the thickness of both ears was measured using vernier calipers (Mitutoyo, Carros, Japan). The weights of ear samples (5 mm in diameter) were measured at the same time.

Hematoxylin-eosin $(H \& E)$ staining. After measuring ear thickness and weight, ear tissues were resected and embedded in paraffin. Sections were then stained with H\&E to observe immune cell infiltration and spongiosis. Stained tissues were

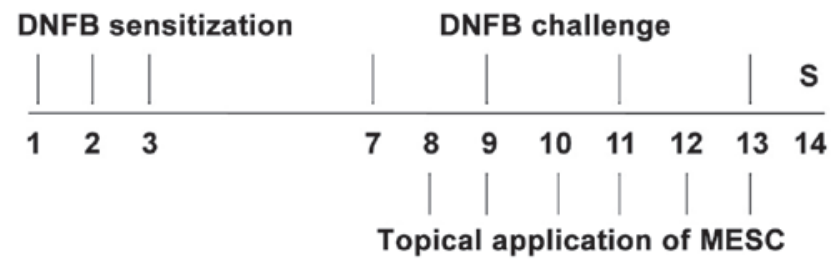

Figure 1. Experimental design. The experimental groups (except for the treatment naïve group) were sensitized by administering DNFB externally to ears daily on days 1 (start), 2 and 3. Mice were challenged with DNFB on days 7, 9, 11 and 13. Animals in the MESC and DEX groups were treated topically with MESC $(30,100$ or $300 \mu \mathrm{g} /$ ear in ethanol and AOO or DEX (75 $\mu \mathrm{g} / \mathrm{ear}$ in ethanol and AOO). All animals were sacrificed on day 14. DNFB, 1-Fluoro-2, 4-dinitrofluorobenzene; MESC, methanol extract of S. chinensis; AOO, acetone: Olive oil; DEX, dexamethasone.

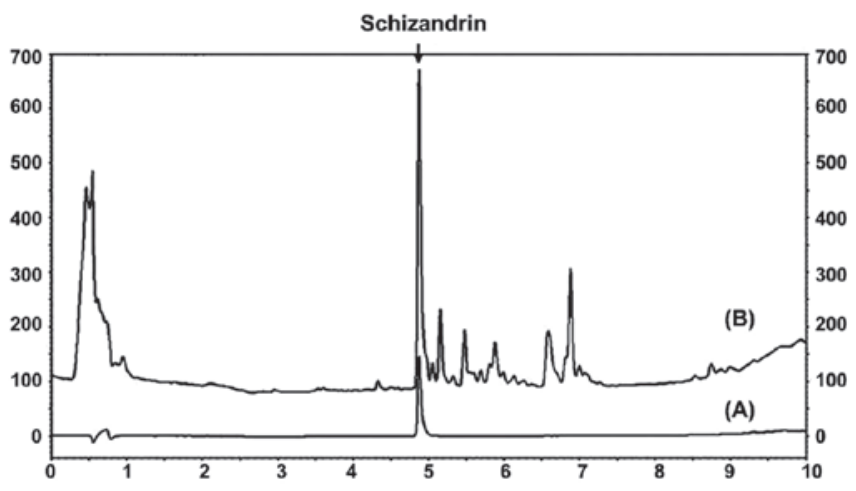

Figure 2. Chromatograms of schizandrin (A) and the MESC (B) obtained at a UV wavelength of $250 \mathrm{~nm}$. MESC, methanol extract of $S$. chinensis.

observed under a light microscope (x100; SZX7; Olympus, Tokyo, Japan).

Measurement of epithelial thickness. H\&E stained slides were thoroughly examined under the light microscope equipped with a digital camera (Coolpix P600; Nikon, Tokyo, Japan), and five images per slide were captured at x200. To measure epithelium thickness, vertical distances between basal lamina and outermost stratum granulosum were measured. Five random measurements were made per slide using Motic Images Plus 2.0 (Motic Instrument Inc., Causeway Bay, Hong Kong).

Evaluation of immune cell infiltration. To evaluate immune cell infiltration, numbers of immune cells in connective tissue were counted in five photographs per slide (x200). Macrophages, polymorphonuclear leucocytes (PMNL), lymphocytes, eosinophils, plasma cells and giant cells were viewed as immune cells.

Measurement of cytokine production. Resected ear tissues were lysed and homogenized with protein extraction solution (Intron Bio) using a bullet blender (Next Advance, Averill Park, NY, USA) to obtain tissue lysates. The levels of tumor necrosis factor (TNF)- $\alpha$, interferon (IFN)- $\gamma$, interleukin-6 (IL-6) and monocyte chemotactic protein-1 (MCP-1) were then measured in $50 \mu \mathrm{g}$ lysate using a cytometric bead array kit (BD Biosciences, San Jose, CA, USA). 

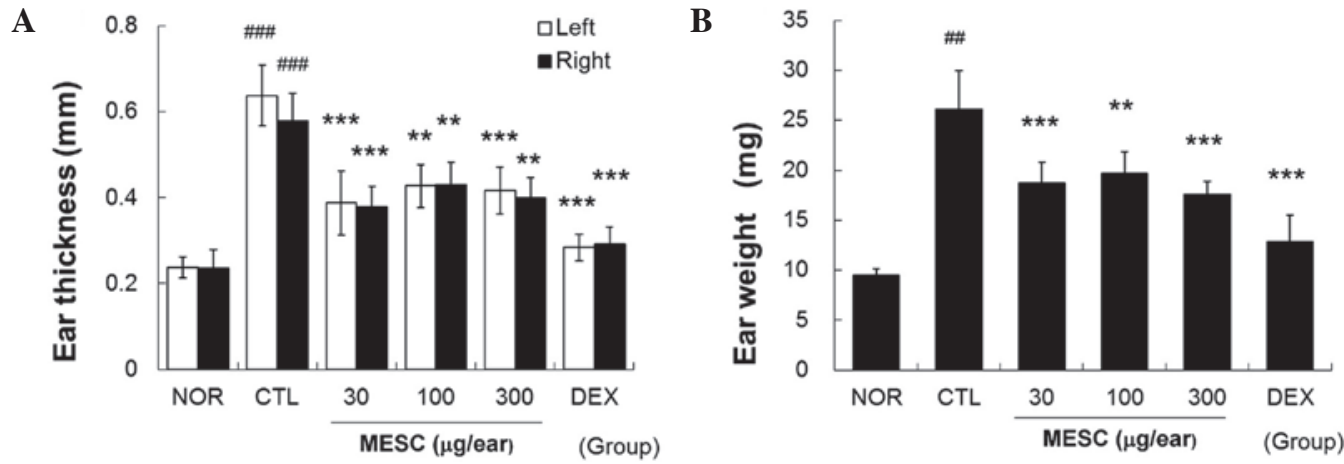

Figure 3. Effects of MESC on ear swelling in mice with CD. Inhibition of ear thickness and body weight in response to the topical application of MESC was analyzed using a vernier caliper and a microbalance on day 14. For 6 days, 30, 100 or $300 \mu \mathrm{g}$ MESC was applied externally onto ears. NOR, treatment naïve mice; CTL, non-treated CD mice; DEX, $75 \mu \mathrm{g} / \mathrm{ear}$ DEX. (A) Ear thickness and (B), ear weight. All values are presented as the mean \pm standard deviation. ${ }^{\# \#} \mathrm{P}<0.01$ and ${ }^{\# \# /} \mathrm{P}<0.001$ vs. NOR; ${ }^{* *} \mathrm{P}<0.01$ and ${ }^{* * *} \mathrm{P}<0.001$ vs. CTL. CD, contact dermatitis; MESC, methanol extract of S. chinensis; DEX, dexamethasone.

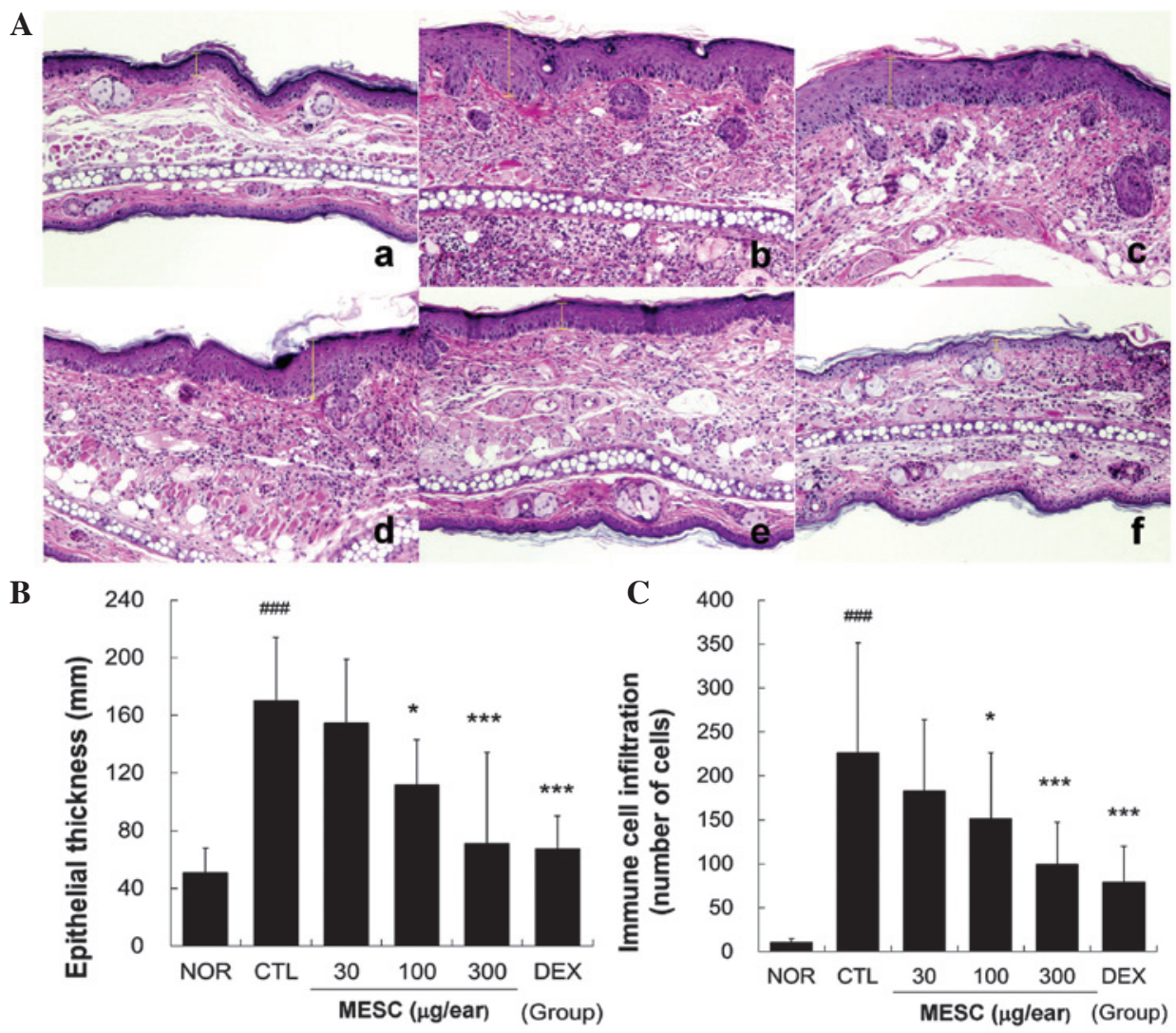

Figure 4. Effects of MESC on histopathological changes in CD mice. (A) Ear tissues were stained with hematoxylin and eosin and observed under a light microscope. (Aa) NOR group; (Ab) CTL group; (Ac) $30 \mu \mathrm{g} /$ ear MESC treated group; (Ad) $100 \mu \mathrm{g} / \mathrm{ear}$ MESC treated group; (Ae) $300 \mu \mathrm{g} / \mathrm{ear}$ MESC treated group; (Af) $75 \mu \mathrm{g} /$ ear DEX treated group. Magnification, x100. The effects on (B) epidermal thickness and (C) immune cell infiltration were also measured in a quantitative manner. CD, contact dermatitis; NOR, treatment naïve mice; CTL, non-treated CD mice; DEX, $75 \mu \mathrm{g} /$ ear of DEX. MESC, methanol extract of S. chinensis; DEX, dexamethasone.

Statistical analysis. The Mann-Whitney test was used for all statistical comparisons, and Prism 5 version 5.01 (GraphPad Software Inc., La Jolla, CA, USA) was used for all analyses. Results are presented as the mean \pm standard deviation. $\mathrm{P}<0.05$ was considered to indicate a statistically significant difference.

\section{Results}

Identification of schizandrin in MESC. The schizandrin peak was detected at a retention time of 4.870 min (Fig. 2).
MESC inhibits increases in ear swelling in CD mice. Repeated application of DNFB increased ear thickness and weight. Marked increases in the thickness and weight of ear tissues were observed in the CTL group. Topical treatment with MESC effectively inhibited increases in thickness and weight, although inhibitory effects were greater in the DEX group than in the MESC group (Fig. 3).

MESC prevents hyperplasia, spongiosis and immune cell infiltration. Repeated application of DNFB induced hyperplasia 

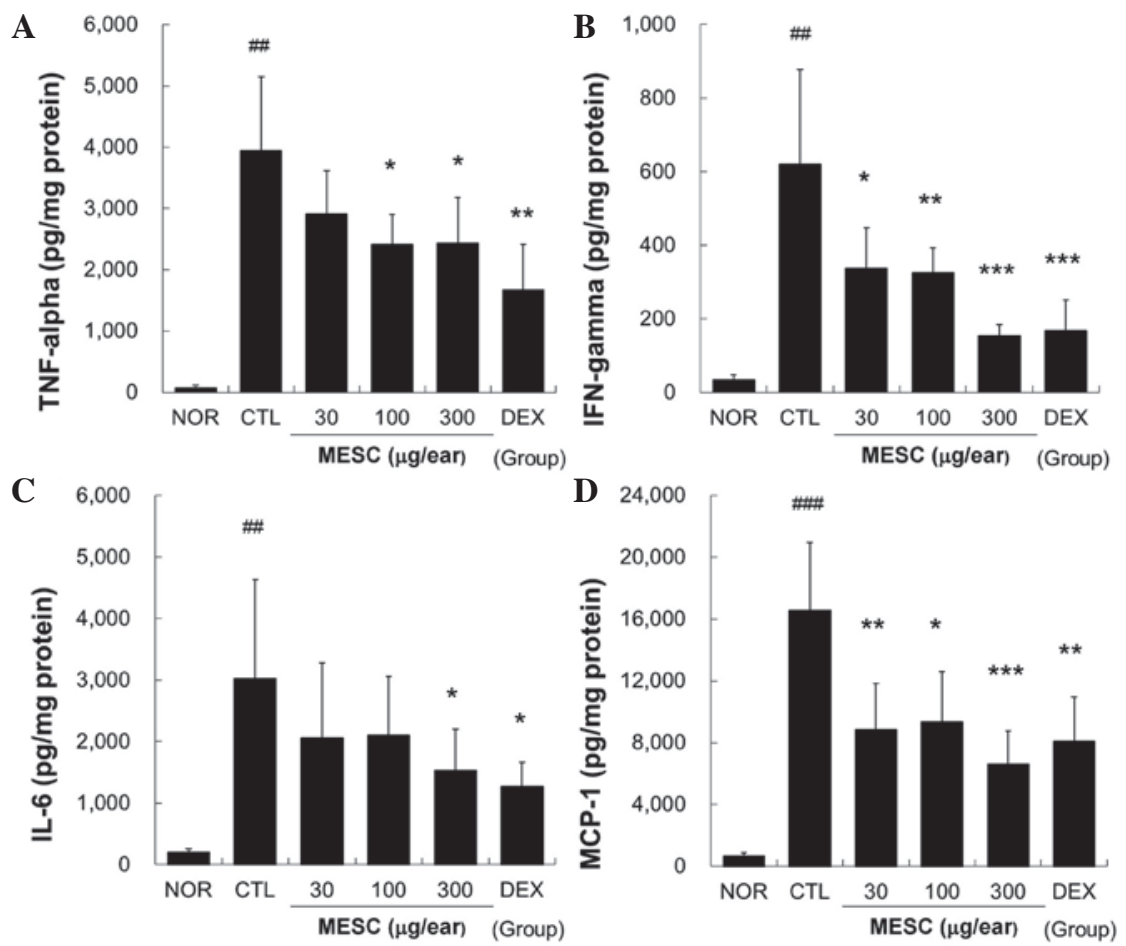

Figure 5. Effects of MESC on cytokine production in mice with CD. Levels of TNF- $\alpha$, IFN- $\gamma$, IL- 6 and MCP-1 in ear tissues were measured using the cytometric bead array method. A total of $50 \mu \mathrm{g}$ tissue lysate was used to measure cytokine levels. (A) TNF- $\alpha$; (B) IFN- $\gamma$; (C) IL-6 and (D) MCP-1. All values are presented as the mean \pm standard deviation. ${ }^{\# \#} \mathrm{P}<0.01$ and ${ }^{\# \# /} \mathrm{P}<0.001$ vs. NOR; ${ }^{*} \mathrm{P}<0.05,{ }^{* *} \mathrm{P}<0.01$ and ${ }^{* * *} \mathrm{P}<0.001$ vs. CTL. NOR, treatment naïve mice; CTL, non-treated CD mice; DEX, $75 \mu \mathrm{g} / \mathrm{ear}$ DEX. CD, contact dermatitis; TNF, tumor necrosis factor; IFN, interferon; IL, interleukin; MCP, monocyte chemoattractant protein; MESC, methanol extract of S. chinensis; DEX, dexamethasone.
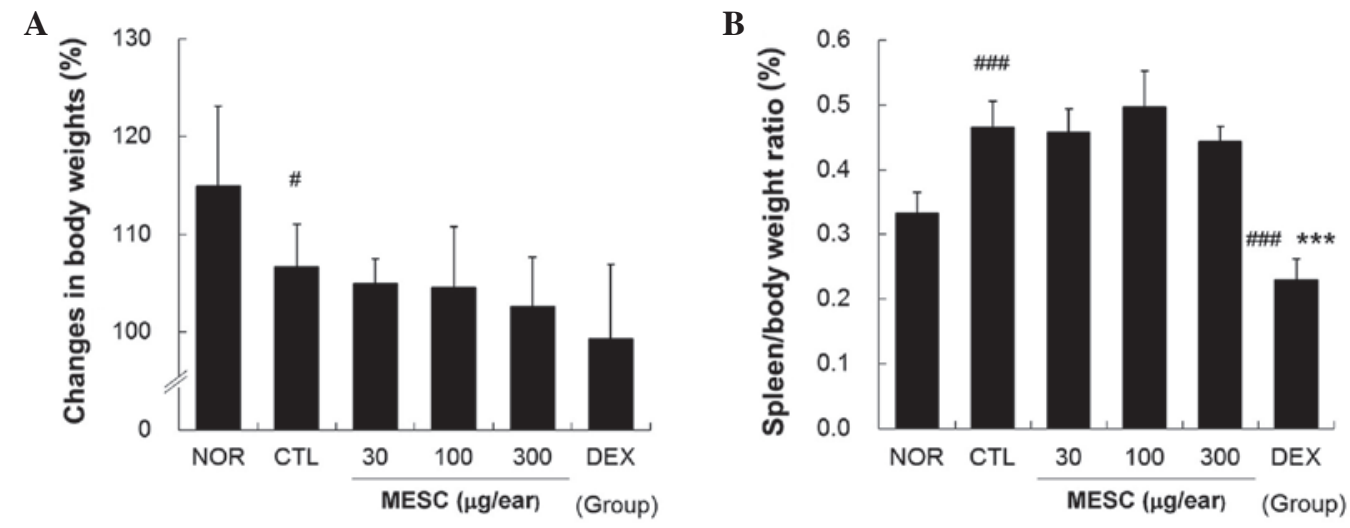

Figure 6. Effects of MESC on body weight gain and spleen/body weight ratio in mice with CD. Body weights were measured on day 1 (experiment start) and 14. (A) Changes in body weights were based on average weights obtained on day 14 and are expressed as percentages of average body weights on day 1. (B) Spleen weights were also measured on day 14, and spleen/body weight ratios were calculated. All values are presented as the mean \pm standard deviation ${ }^{\#} \mathrm{P}<0.05$ and ${ }^{\# \# \#} \mathrm{P}<0.001$ vs. NOR; ${ }^{* * *} \mathrm{P}<0.001$ vs. CTL. NOR, treatment naïve mice; CTL, non-treated CD mice; DEX, $75 \mu \mathrm{g} /$ ear DEX. CD, contact dermatitis; MESC, methanol extract of S. chinensis; DEX, dexamethasone.

and significant edema and spongiosis, which are the hallmarks of skin inflammation. Marked immune cell infiltration was observed in the CTL group, whereas little hyperplasia, edema or spongiosis was observed in the MESC group. Treatment with $30 \mu \mathrm{g} /$ ear MESC had a marginal effect on hyperplasia and immune cell infiltration. However, treatment with DEX more effectively prevented spongiosis, hyperplasia and immune cell infiltration (Fig. 4).

MESC reduces the levels of TNF- $\alpha, I F N-\gamma, I L-6$ and MCP-1 in the ear tissues of $C D$ mice. Marked increases in TNF- $\alpha$,
IFN- $\gamma$, IL- 6 and MCP-1 levels were observed in the CTL group, but these increases in TNF- $\alpha$, IFN- $\gamma$, IL- 6 and MCP- 1 levels were effectively reduced by topical MESC. Treatment with MESC at $<100 \mu \mathrm{g} /$ ear did not affect IL-6 levels (Fig. 5).

MESC does not affect spleen/body weight ratio in CD mice. Body weight gains in the CTL group were lower than in the NOR group (Fig. 6A), whereas no body weight gains were observed in the MESC or DEX groups (Fig. 6A). The effects of MESC on spleen enlargement were estimated by determining spleen/body weight ratios, which were significantly smaller in 
the DEX group than in the CTL group, but almost the same in the MESC and CTL groups (Fig. 6B).

\section{Discussion}

Inflammation is mediated by a variety of soluble factors, including a group of secreted polypeptides termed cytokines (12). Several cytokines are important in mediating acute or chronic inflammatory reactions, such as IFN- $\gamma$, IL-6, MCP-1 and TNF- $\alpha$ (13). The cytokines known to mediate chronic inflammatory processes can be divided into those participating in humoral inflammation, such as IL-6, and those contributing to cellular inflammation, such as IFN- $\gamma$ and TNF- $\alpha$ (13). In the present study, MESC effectively reduced immune cell infiltration into affected tissues and reduced pro-inflammatory cytokine production. These results suggest that MESC acts as an anti-inflammatory agent and that the major mechanism underlying its effects involves suppression of pro-inflammatory cytokine production.

S. chinensis Turcz. fruit is a well-known traditional Korean herbal medicine and is used as an antitussive and sedative agent, as well as to improve liver function in patients with viral hepatitis (14). Furthermore, it has been shown to have anti-oxidative, antimicrobial and nitrite scavenging effects (15). Yasukawa et al (16) concluded that S. chinensis (gomesin A) exhibits anti-inflammatory activity, and found that it inhibited tumor promotion by 12-O-tetradecanoylphorbol-13-acetate in a mouse skin model of carcinogenesis. Huyke et al (17) suggested that $S$. chinensis may be useful for the prevention and treatment of hyperproliferative and inflammatory skin diseases, whereas Kang et al (18) reported that $S$. chinensis inhibited the secretion of pro-inflammatory cytokines by mast cells. These cells contain potent inflammatory mediators, such as histamine and multifunctional cytokines, which can promote the production of pro-inflammatory cytokines, adhesion molecules and growth factors in resident cells (19). In the present study, MESC reduced TNF- $\alpha$, IFN- $\gamma$, IL- 6 and MCP-1 levels, showing that its anti-inflammatory effects are attributable to cytokine regulation.

In the present study, DEX reduced body weight gain and spleen/body weight ratios. Spleen/body weight ratios in the DEX group were significantly lower than in the NOR group. By contrast, MESC did not affect body weight gains or spleen/ body weight ratios. In a previous study by Nagao et al (20), it was reported that corticosteroid-induced weight loss was the result of adverse reactions. In addition, the spleen mass reduction observed in the DEX group could have reflected general immune suppression, which is one of the major side effects of corticosteroids.

This study reports the anti-inflammatory effects of MESC on CD in vivo. MESC effectively prevented ear swelling, hyperplasia, spongiosis and the infiltration of immune cells into inflamed tissues. In addition, levels of TNF- $\alpha$, IFN- $\gamma$ and IL- 6 in affected tissues were dose-dependently reduced by MESC. These results suggest that MESC can effectively prevent inflammatory reactions, and that it should be considered as a complementary or alternative treatment for patients with inflammatory skin diseases.

\section{Acknowledgements}

This study was supported by the National Research Foundation of Korea funded by the Korean government (grant no. 2014R1A5A2009936).

\section{References}

1. Huang T, Shen P and Shen Y: Preparative separation and purification of deoxyschisandrin and gamma-schisandrin from Schisandra chinensis (Turcz) Baill by high-speed counter-current chromatography. J Chromatogr A 1066: 239-242, 2005.

2. Panossian A and Wikman G: Pharmacology of Schisandra chinensis Bail: an overview of Russian research and uses in medicine. J Ethnopharmacol 118: 183-212, 2008.

3. Kang YH and Shin HM: Inhibitory effects of Schizandra chinensis extract on atopic dermatitis in NC/Nga mice. Immunopharmacol Immunotoxicol 34: 292-298, 2012;

4. Chiu PY, Lam PY, Yan CW and Ko KM: Schisandrin B protects against solar irradiation-induced oxidative injury in BJ human fibroblasts. Fitoterapia 82: 682-691, 2011.

5. Lam PY, Yan CW, Chiu PY, Leung HY and Ko KM: Schisandrin B protects against solar irradiation-induced oxidative stress in rat skin tissue. Fitoterapia 82: 393-400, 2011.

6. Kang JI, Kim SC, Hyun JH, Kang JH, Park DB, Lee YJ, Yoo ES and Kang HK: Promotion effect of Schisandra nigra on the growth of hair. Eur J Dermatol 19: 119-125, 2009.

7. Liu C, Zhang S and Wu H: Non-thermal extraction of effective ingredients from Schisandra chinensis Baill and the antioxidant activity of its extract. Nat Prod Res 23: 1390-1401, 2009.

8. Guo LY, Hung TM, Bae KH, Shin EM, Zhou HY, Hong YN, Kang SS, Kim HP and Kim YS: Anti-inflammatory effects of schisandrin isolated from the fruit of Schisandra chinensis Baill. Eur J Pharmacol 591: 293-299, 2008.

9. Diepgen TL: Occupational skin diseases. J Dtsch Dermatol Ges 10: 297-313, 2012.

10. Cohen DE and Heidary N: Treatment of irritant and allergic contact dermatitis. Dermatol Ther 17: 334-340, 2004.

11. Park HH, Lee JS, Yun H, Hwang G and Chong MS: Effect of Schisandrae chinensis fructus on keratinocyte damage by UV irradiation. Korean J Oriental Physiology and Pathology 26: 330-337, 2012.

12. Oskeritzian CA: Mast cell plasticity and sphingosine-1-phosphate in immunity, inflammation and cancer. Mol Immunol 63: 104-112, 2015.

13. Sebastiani S, Albanesi C, De PO, Puddu P, Cavani A and Girolomoni $\mathrm{G}$ : The role of chemokines in allergic contact dermatitis. Arch Dermatol Res 293: 552-559, 2002.

14. Ou M: China-English manual of common-used prescriptions in traditional Chinese medicine. Guangdong Science and Technology Press, Guangzhou, China. 69-70, 1992.

15. Jung GT, Ju IO, Choi JS and Hong JS: The antioxidative, antimicrobial and nitrite scavenging effects of Schisandra chinensis RUPRECHT (Omija) seed. Korean J Food Sci Technol 32: 928-935, 2000

16. Yasukawa K, Ikeya Y, Mitsuhashi H, Iwasaki M, Aburada M, Nakagawa S, Takeuchi M and Takido M: Gomisin A inhibits tumor promotion by $12-\mathrm{O}$-tetradecanoylphorbol-13-acetate in two-stage carcinogenesis in mouse skin. Oncology 49: 68-71, 1992.

17. Huyke C, Engel K, Simon-Haarhaus B, Quirin KW and Schempp CM: Composition and biological activity of different extracts from Schisandra sphenanthera and Schisandra chinensis. Planta Med 73: 1116-1126, 2007.

18. Kang OH, Chae HS, Choi JH, Choi HJ, Park PS, Cho SH, Lee GH, So HY, Choo YK, Kweon OH and Kwon DY: Effects of the Schisandra fructus water extract on cytokine release from a human mast cell line. J Med Food 9: 480-486, 2006.

19. Kanda $\mathrm{N}$ and Watanabe $\mathrm{S}$ : Histamine enhances the production of granulocyte-macrophage colony-stimulating factor via protein kinase Calpha and extracellular signal-regulated kinase in human keratinocytes. J Invest Dermatol 122: 863-872, 2004.

20. Nagao K, Akabane H, Masuda T, Komai M, Tanaka H and Nagai H: Effect of MX-68 on airway inflammation and hyperresponsiveness in mice and guinea-pigs. J Pharm Pharmacol 56: 187-196, 2004. 